Study of bandgap property of a bilayer membrane-type metamaterial applied on a thin plate

Cong GAO, Dunant HALIM, Xiaosu Yi

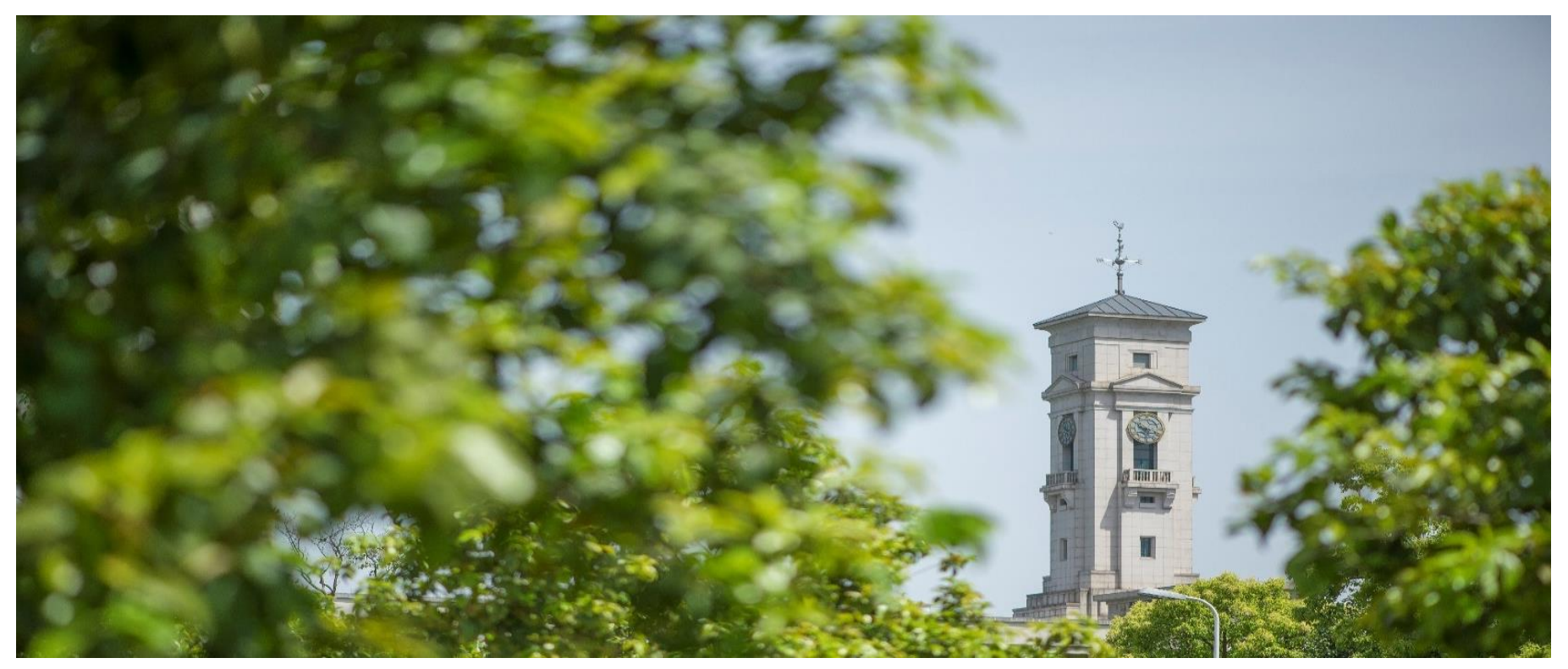


Faculty of Science and Engineering, University of Nottingham Ningbo China, 199 Taikang East Road, Ningbo, 315100, Zhejiang, China.

First published 2020

This work is made available under the terms of the Creative Commons Attribution 4.0 International License:

http://creativecommons.org/licenses/by/4.0

The work is licenced to the University of Nottingham Ningbo China under the Global University Publication Licence:

https://www.nottingham.edu.cn/en/library/documents/researchsupport/global-university-publications-licence.pdf 


\title{
Study of bandgap property of a bilayer membrane-type metamaterial applied on a thin plate
}

\author{
Cong $\mathrm{GAO}^{1}$, Dunant HALIM*, Xiaosu $\mathrm{Yi}^{2}$ \\ ${ }^{1}$ International Doctoral Innovation Centre, University of Nottingham Ningbo China, 199 Taikang \\ East Road, Ningbo 315100, China \\ *International Doctoral Innovation Centre, Department of Mechanical, Materials and \\ Manufacturing Engineering, University of Nottingham Ningbo China, 199 Taikang East Road, \\ Ningbo 315100, China
}

${ }^{2}$ Department of Mechanical, Materials and Manufacturing Engineering, University of Nottingham Ningbo China, 199 Taikang East Road, Ningbo 315100, China

\begin{abstract}
This work is aimed to study the bandgap property of a thin plate structure with periodically attached bilayer membrane-type resonators. An analytical method based on the Plane Wave Expansion (PWE) method combined with the Rayleigh method, is proposed to predict the bandgap property of bilayer membrane-type metamaterials. The accuracy of the proposed method is verified by the finite element analysis, and a parametric analysis is conducted to reveal the effect of parameters on the bandgap performance. It is found that such a metamaterial can generate two separated bandgaps through the contribution of its two layers of membranes. It is observed that the increase of membrane tensile stress or the magnitude of attached mass can lead to the broadening of bandgaps, whilst the change of unit cell's periodicity has the opposite effect. In addition, if compared with the corresponding single layer membrane-type metamaterials, it is shown that the bilayer membranetype's first bandgap is suppressed while the second one is extended. However, by applying proper membrane tensile stress and mass magnitude, the suppression of the first bandgap can be weakened whilst allowing the tuning of the bandgap location. These characteristics reveal the benefits of using bilayer membrane-type metamaterial as it possesses higher agility in bandgap tuning. The proposed method can provide an effective tool for the bilayer membrane-type metamaterial design and optimisation.
\end{abstract}

Keywords: membrane-type metamaterial; bilayer membrane-type resonator; bandgap property; vibration absorption.

\section{Introduction}

Phononic crystals and acoustic metamaterials are manually engineered structures that can generate bandgaps over certain frequency regions within which the propagation of elastic and acoustic waves can be manipulated $[1,2,3,4]$. Phononic crystals are formed by periodically located unit cells in a certain lattice constant and as a result, the formation of bandgap in a phononic crystal is mainly attributed to the Bragg scattering [5]. Under this mechanism, it requires the unit cell's lattice constant to be in the same order of magnitude of the wavelength. Consequently, the structure size has to be large when it is utilized for application in low-frequency regions. The study undertaken by Liu et al. [6] broke this phononic crystal's limitation by proposing an acoustic metamaterial that can generate bandgap through a localized resonance phenomenon. This type of metamaterial is composed of unit cells that can function as independent resonators [7]. When the incident wave's frequency is close to the resonant frequency of the unit cells, the resonance modes will be excited and absorb most of the vibration energy and store it within the cells. Thus the vibration of the 
primary structure is suppressed. Since then, an increasing amount of literature has investigated the locally resonant type metamaterial and various designs have been proposed $[8,9,10]$. As the triggering of locally resonant only depends on the incident frequency, it can thus ignore the Rayleigh scattering barrier and control the wave propagation in subwavelength structure sizes [11].

In the acoustic metamaterial field, metamaterial designs have been proposed to achieve satisfactory sound isolation or absorption performances [12, 13, 14]. In 2008, Yang et al. [15] published a paper in which they proposed a membrane-type acoustic metamaterial (MAM) for sound isolation purposes. The MAMs are formed by low surface mass density unit cells. A unit cell is composed of a pre-stressed elastic membrane with attached mass platelet, and a rigid frame on which the membrane is fixed [16]. This particular configuration has the advantages of being light-weight, easy to manufacture and relatively low cost. Such a design enables the MAMs to possess sound absorption and isolation capabilities despite the mass-law limitation [17, 18, 19, 20].

However, most studies on MAMs were primarily focused on sound isolation or absorption applications, with a limited number of studies on vibration control applications. Sun et al. [21] investigated the membrane-type resonator's performance as a tuned mass damper. The research work indicated that by attaching two membrane-type resonators to an aluminium beam, the structural vibration can be attenuated effectively. Sun [22] proposed a membrane-type sample with multiple mass platelets attached on a rectangular membrane and experimentally demonstrated that this structure possessed a better vibration damping performance than a commercial damping material. These research works revealed the feasibility of using membrane-type resonators for structural vibration control applications. However, there are still areas that need further investigation, such as the effect of membrane-type resonator's parameters (e.g. material property, membrane tensile stress, the magnitude of attached mass) on the vibration absorption performance, and methods for fast prediction of MAMs' bandgap property.

In addition to studies focused on single layer MAMs, some researches have been undertaken for investigating the use of MAMs with bilayer membranes, and it was found that bilayer structures can achieve a better sound isolation performance than a single layer type [23, 24]. A multiple layered membrane-type metamaterial can generate a number of bandgaps. In order to achieve an enhanced vibration control performance, these bandgaps can be merged into a large continuous one by proper parameter tuning [25]. Multiple layered MAMs can be easily formed by stacking several single layer MAMs and it requires no extra occupied area on the primary structure.

Moreover, it is observed that the bandgap mechanism of a MAM mainly depends on the fundamental mode resonance of the resonators [26]. Thus, the prediction of membrane structure's fundamental resonant frequency will be a key design factor for MAMs. There were several methods proposed for the calculation of the membrane-mass model's resonant frequencies [27, 28]. However, limited studies have focused on the prediction of membrane-type metamaterial's bandgap property in terms of the bandgap location and width. Chen et al. [29] introduced a theoretical model for predicting the sound absorption performance of MAM. The model based on a vibroacoustic plate model in conjunction with the point matching method and it can conduct accurate prediction for the resonant frequencies. Tensile stress applied to the membrane structure was not considered as an independent design parameter. When designing and optimising a MAM for a particular application, the prediction of the design parameters' effects on the bandgap property is essential. Commonly, it is achieved by undertaking the finite element analysis (FEA) [30] on MAMs. However, the FEA-based optimisation process for optimising the design parameters can be computationally costly and time-consuming. In contrast, the use of an analytical model allows optimisation of design parameters to be more rapidly and convenient.

Hence, in this work, an analytical model that can predict the bandgap property of the composite meta-structure is proposed. It provides a convenient optimisation tool that allows rapid predictions 
of the bandgap location and width. In contrast with the previous work that mainly focused on the prediction of bandgap location, the proposed model could be used to predict both bandgap width and location. It incorporates the design parameters as independent variables so it is convenient to study their effects on the bandgap property. This study allows the development of design guidelines for the proposed membrane-type metamaterials for vibration control applications.

The paper is structured as follows: Section 2 introduces the structure of the membrane-type metamaterial and presents its theoretical background. Section 3 presents the bandgap of an infinite metamaterial structure and the effects of different design parameters to the vibration control performance. Section 4 illustrates the FEA simulation results that validate the accuracy of the proposed method, while Section 5 provides the conclusions of the work.

\section{The modelling of bilayer membrane-type resonators}

In this work, a model of bilayer resonator attached to a thin plate is employed. The model is schematically depicted in Figure 1. Two single layer membrane-type resonators are stacked together to form a bilayer membrane-type resonator. Hollows are made on the side of the frame so to minimize the effect of air loading when it is assembled. The elastic membrane is pre-stressed and fixed onto the upper surface of the rigid frame whilst a mass is attached at the centre of the membrane. The materials of membrane, mass and frame are chosen to be silicon rubber, copper and epoxy, respectively.

The geometrical parameters and material property of the membrane-type resonators and the primary plate structure are given in Table 1 . For a square frame, the sides lengths are defined as $a_{1}$ $=a_{2}=a$. Since the bilayer membrane-type resonators are attached periodically onto the thin plate, the lattice constant of the formed metastructure equals to side length $a$.

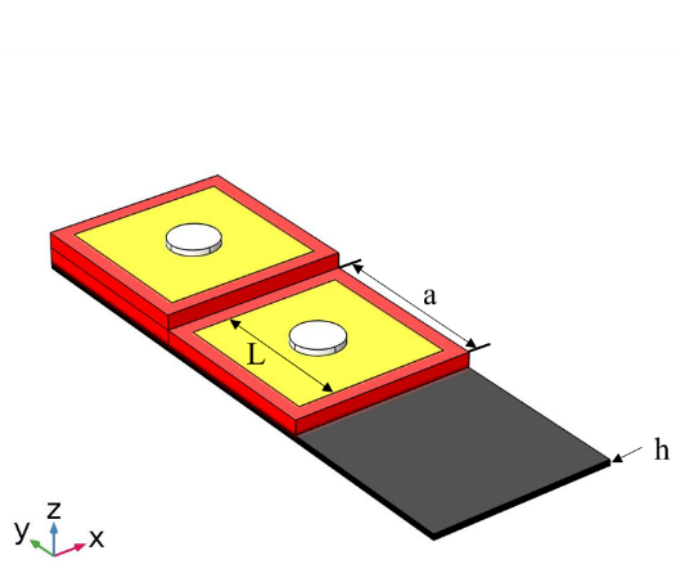

(a)

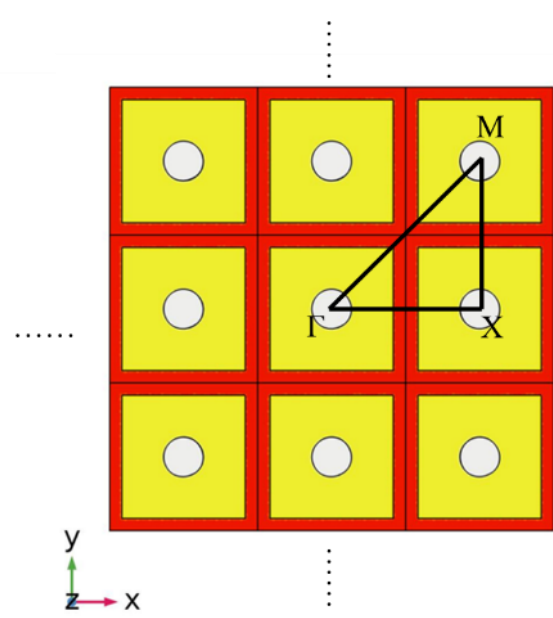

(b)

Figure 1. (a) The configuration of stacked membrane-type resonators attached to a thin plate; (b) The periodic structure of the metamaterial plate and its corresponding Brillouin zone. The membranes, masses, frames and the thin plate are represented by yellow, white, red and black colours respectively. 


\begin{tabular}{cccccc}
\hline Membrane & \multicolumn{3}{c}{ Frame } & \multicolumn{2}{c}{ Plate } \\
\hline Side length $L(\mathrm{~mm})$ & 50 & Side length $a(\mathrm{~mm})$ & 60 & Thickness $(\mathrm{mm})$ & 2 \\
Thickness $t(\mathrm{~mm})$ & 0.1 & Height $H(\mathrm{~mm})$ & 5 & $E(\mathrm{GPa})$ & 70 \\
$E(\mathrm{MPa})$ & 1.9 & $E(\mathrm{GPa})$ & 2.65 & Poisson's ratio & 0.33 \\
Poisson's ratio & 0.48 & Poisson's ratio & 0.41 & Density $\left(\mathrm{kg} / \mathrm{m}^{3}\right)$ & 2700 \\
Density $\left(\mathrm{kg} / \mathrm{m}^{3}\right)$ & 980 & Density $\left(\mathrm{kg} / \mathrm{m}^{3}\right)$ & 1100 & Table 1: The geometrical parameters of membrane-type resonators
\end{tabular}

\subsection{The estimation of equivalent stiffness for a membrane-type resonator system}

The membrane-type resonator's structure can be simplified to a mass-spring model. Within the resonator, evenly biaxial tensile stress $T$ is applied to the membrane with the assumption that the stress within the membrane is uniformly distributed [31]. The equivalent stiffness is contributed by tensile stress applied to the membrane.

The equivalent stiffness of the system is estimated by using the Rayleigh method [26] through the estimation of the fundamental resonant frequency. For a rectangular membrane with side lengths of $L_{1}$ and $L_{2}$, the attached mass is assumed in the form of a concentrated point mass, located at coordinates $(a, b)$ over the membrane. The strain energy $E_{s}$ and kinetic energy $E_{k}$ of the resonator can be respectively expressed as:

$$
\begin{gathered}
E_{s}=\frac{1}{2} \iint D\left\{\left(\frac{\partial^{2} w}{\partial x^{2}}+\frac{\partial^{2} w}{\partial y^{2}}\right)^{2}-2(1-v)\left[\frac{\partial^{2} w}{\partial x^{2}} \frac{\partial^{2} w}{\partial y^{2}}-\left(\frac{\partial^{2} w}{\partial x \partial y}\right)^{2}\right]\right\} d x d y \\
+\frac{1}{2} \iint T\left[\left(\frac{\partial^{2} w}{\partial x^{2}}\right)^{2}+\left(\frac{\partial^{2} w}{\partial y^{2}}\right)^{2}\right] d x d y \\
E_{k}=\frac{\omega^{2}}{2}\left\{\iint m_{s} w^{2}(x, y) d x d y+M(a, b) w^{2}(a, b)\right\}
\end{gathered}
$$

where $D=\frac{E t^{3}}{12\left(1-v^{2}\right)}$ is the bending stiffness of the membrane; $E, t$ and $v$ are the respective Young's modulus, thickness and Poisson's ratio of the membrane; $m_{s}$ is the membrane mass per unit area; $T$ is the membrane tensile stress per unit length; $M(a, b)$ is the mass located at coordinates $(a, b) ; w(x, y)$ and $w(a, b)$ are the respective transverse displacements of the membrane and mass, and $\omega$ is the frequency.

For a membrane-type resonator, the bandgap is normally influenced by the fundamental resonant frequency [26]. In this case, the mode shape function for this particular model can be estimated using a doubly-sine function [26]:

$$
w(x, y)=A_{m n} \sin \left(\frac{\pi x}{L_{1}}\right) \sin \left(\frac{m \pi x}{L_{2}}\right) \sin \left(\frac{\pi y}{L_{1}}\right) \sin \left(\frac{n \pi y}{L_{2}}\right) .
$$

Based on equations (1) - (3), the fundamental resonant frequency is obtained as:

$$
\omega_{11}=\sqrt{\frac{\frac{\pi^{4} D}{4 L_{1}{ }^{3} L_{2}{ }^{3}}\left(3 L_{1}{ }^{4}+3 L_{2}{ }^{4}+2 L_{1}{ }^{2} L_{2}{ }^{2}\right)+\frac{3\left(L_{1}{ }^{2}+L_{2}{ }^{2}\right) T \pi^{2}}{16 L_{1} L_{2}}}{\frac{9 L_{1} L_{2} m_{S}}{64}+M \sin ^{4}\left(\frac{\pi a}{L_{1}}\right) \sin ^{4}\left(\frac{\pi b}{L_{2}}\right)}} .
$$

Equation (4) describes the fundamental resonant frequency of a membrane-type resonator. This method has been proved to be feasible in predicting the resonant frequency of a membrane-type 
resonator $[26,28]$. Considering the resonators as a single degree of freedom mass-spring system, the effective stiffness $k_{e}$ can therefore be described by:

$$
k_{e}=\omega_{11}^{2} M
$$

\subsection{The dispersion relation of the metamaterial unit cell}

The attached membrane-type resonator and the corresponding part of the plate are considered as a unit cell of the metastructure. Its bandgap behaviour can be expressed by the dispersion relation of a unit cell with infinite periodic boundary conditions. The thin plate structure can be simplified as the configuration shown in Figure 2. Since there are two layers of membranes, two resonators are attached in each unit cell. For a rectangular lattice structure, the lattice constants are defined as $a_{1}$ and $a_{2}$.

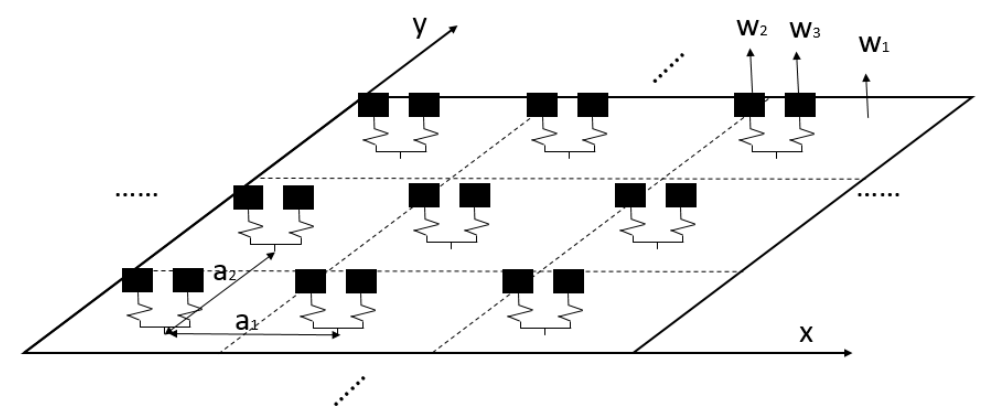

Figure 2. A configuration of thin plate with periodically attached spring-mass resonators.

For a periodic structure, the coordinates of the resonators attached points on the plate can be replaced by the lattice vector as:

$$
R=p a_{1}+q a_{2}
$$

where $p$ and $\mathrm{q}$ are integers that represent the unit distances of the resonator to the original point. Replacing the coordinates by the lattice vectors, the system's equations of motion can be described as:

$$
\left\{\begin{array}{c}
D \nabla^{4} w_{1}(r)-\omega^{2} \rho_{p} h w_{1}(r)=\sum_{R}\left[f_{1}(R) \delta(r-R)\right]+\sum_{R}\left[f_{2}\left(R^{\prime}\right) \delta\left(r-R^{\prime}\right)\right] \\
-\omega^{2} m_{R 1} w_{2}(R)=f_{m 1}(R) \\
-\omega^{2} m_{R 2} w_{3}\left(R^{\prime}\right)=f_{m 2}\left(R^{\prime}\right) .
\end{array}\right.
$$

where $\mathrm{r}$ and $\mathrm{R}$ represent the location of points on the plate and the location of resonators, respectively; $D=\frac{E h^{3}}{12\left(1-v^{2}\right)}$ is the flexural stiffness of the plate; $\rho_{p}$ is the mass density of the plate, $m_{R 1}$ and $m_{R 2}$ are resonator masses, respectively; $h$ is the thickness of the plate; $w_{1}(r), w_{2}(R)$ and $w_{3}\left(R^{\prime}\right)$ are respective transverse displacements of plate and two resonators at different points; $f_{1}$ and $f_{2}$ are forces applied on thin plate by resonators; $f_{m 1}$ and $f_{m 2}$ are forces applied on the resonators; and $\delta$ is the Dirac function.

With a bilayer membrane-type resonator, the locations of two resonators can be assumed to be the same, so $R=R^{\prime}$ and:

$$
\begin{aligned}
& f_{1}(R)=-f_{m 1}(R)=-k_{R 1}\left[w_{1}(R)-w_{2}(R)\right] \\
& f_{2}(R)=-f_{m 2}(R)=-k_{R 2}\left[w_{1}(R)-w_{3}(R)\right]
\end{aligned}
$$


For a periodic structure, the Bloch theorem can be adopted and the displacements can be represented by:

$$
\begin{gathered}
w_{1}(r)=\sum_{G}\left[W_{1}(G) e^{-i(k+G) r}\right] \\
w_{i}(R)=w_{i}(0) e^{-i k R}(i=1,2,3 .)
\end{gathered}
$$

where $k=\left(k_{x}, k_{y}\right)$ and $G=p b_{1}+q b_{2}$ are the Bloch wave vector and reciprocal-lattice vector respectively.

By eliminating the Dirac function using the process described in [7], the Dirac function part of the equation can be described by:

$$
\sum_{R} e^{-i k R} \delta(r-R)=e^{-i k r} \sum_{R} \delta(r-R)=e^{-i k r} \sum_{G} \frac{1}{S} e^{-i G r}=\frac{1}{S} \sum_{G} e^{-i(k+G) r} .
$$

Substituting equations (8) - (12) into equation (7) gives:

$$
\left\{\begin{aligned}
D\left[(k+G)_{x}^{2}+(k+G)_{y}^{2}\right]^{2} W_{1}(G)-\omega^{2} \rho_{p} h W_{1}(G) & =-\frac{k_{R 1}}{S} \sum_{G}\left[W_{1}(G)-W_{2}(0)\right]-\frac{k_{R 2}}{S} \sum_{G}\left[W_{1}(G)-W_{3}(0)\right] \\
-\omega^{2} m_{R 1} W_{2}(0) & =k_{R 1} \sum_{G}\left[W_{1}(G)-W_{2}(0)\right] \\
-\omega^{2} m_{R 2} W_{3}(0) & =k_{R 2} \sum_{G}\left[W_{1}(G)-W_{3}(0)\right]
\end{aligned}\right.
$$

Equations (13) describe the governing equation of motion of an infinite plate structure, where it originally requires an infinite superposition of plane waves. However, similar to the mode superposition method, the use of the first few plane wave numbers is already sufficient to achieve accurate results [32]. In this work, therefore, the first 5 wavenumbers are used, which has been observed to be able to generate accurate results.

Equation (13) can then be expressed in matrix form as:

$$
\left(\left[\begin{array}{ccc}
D S[K]+k_{R 1}[U]+k_{R 2}[U] & -k_{R 1}[P] & -k_{R 2}[P] \\
-k_{R 1}\left[P^{T}\right] & k_{R 1} & 0 \\
-k_{R 2}\left[P^{T}\right] & 0 & k_{R 2}
\end{array}\right]-\omega^{2}\left[\begin{array}{ccc}
\rho_{p} h S[I] & \mathbf{0} & \mathbf{0} \\
0 & m_{R 1} & 0 \\
0 & 0 & m_{R 2}
\end{array}\right]\right) \times\left[\begin{array}{c}
W_{1} \\
w_{2}(0) \\
w_{3}(0)
\end{array}\right]=0
$$

The dispersion relation can then be derived by solving the eigenvalue problem of the matrix equation (14), which a commonly used PWE method for calculating bandgap property of phononic crystals $[33,34]$.

\section{The bandgap property of an infinite membrane-type metamaterial structure}

\subsection{The band structure of the membrane-type metamaterial}

Based on the developed model, an investigation on the bandgap property of an infinite bilayer membrane-type metamaterial structure is conducted. Define the masses attached to both membranes as $m_{R 1}=m_{R 2}=2.0 \mathrm{~g}$, and the stresses applied to the upper and lower membrane layer as $0.6 \mathrm{MPa}$ and $0.8 \mathrm{MPa}$, respectively. For $a=60 \mathrm{~mm}$ and plate thickness of $h=2.0 \mathrm{~mm}$, the mass of the bottom aluminium plate area is $m_{1}=19.4 \mathrm{~g}$. According to the content in Section 2, the respective effective stiffness of the two resonators can be obtained as $k_{R 1}=217.4 \mathrm{~N} / \mathrm{m}$ and $k_{R 2}=$ $290.6 \mathrm{~N} / \mathrm{m}$. The formation of the bandgap depends on the activation of the local resonant modes 
of the metamaterial [35]. If flexural waves and longitudinal waves can activate resonant modes in a metamaterial respectively, then stop bands for both types of waves can exist simultaneously [36]. In the bilayer membrane-type resonator, however, the main resonant modes of membrane layers are associated with their out-of-plane displacements, in contrast to in-plane displacements. In this case, these out-of-plane modes of the resonators can be effectively activated by propagating flexural waves in the structure. Since this work is aimed to regulate vibration of a thin plate at low frequencies, which are dominated by flexural vibration, this study investigated the flexural wave stop bands, instead of longitudinal wave ones.

Figure 3 shows that there are two clear flexural bandgaps at: (1) $52.5-54.3 \mathrm{~Hz}$; (2) $60.7-64.5 \mathrm{~Hz}$. When the incident wave's frequency lies within one of the bandgaps, the corresponding resonance of the resonator will be excited, leading to the attenuation of the propagated wave [37].

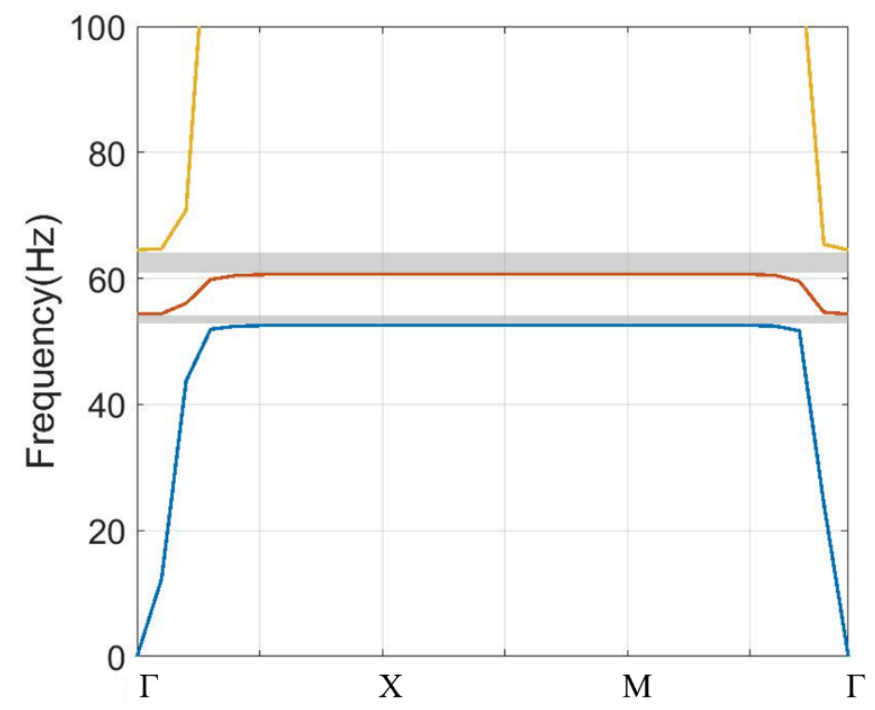

Figure 3. The bandgap structure of a bilayer membrane-type metamaterial with membranes' tensile stresses of 0.6MPa and $0.8 \mathrm{MPa}$, respectively. Two full bandgaps (shaded areas) exist at $52.5-54.3 \mathrm{~Hz}$ and $60.7-64.5 \mathrm{~Hz}$.

By applying different membrane tensile stress in the two resonators, a bilayer membrane-type metamaterial can thus be utilized to control structural vibration in two separated frequency ranges, albeit in relatively narrow operation frequency regions. As shown in the figure, the red curve, which associated with the fundamental resonant frequency of the resonator with $0.8 \mathrm{MPa}$ tensile stress, separates the two full bandgaps.

\subsection{Effects of design parameters on the bandgap property}

In this work, the magnitude of attached masses of resonators, the level of applied tensile stress of membranes, and the periodicity of resonators are studied as the key design factors. The effects of design parameters on the bandgap property are investigated here by using the proposed theoretical model.

\subsubsection{The magnitude of attached masses}

In this subsection, the effect of tuning the attached mass magnitudes are investigated by the proposed analytical model. Figure 4(a) presents the change in the bandgap width and location for a bilayer metamaterial when the magnitudes of both masses $m_{R 1}$ and $m_{R 2}$ are simultaneously varied from $2.0 \mathrm{~g}$ to $60.0 \mathrm{~g}$. Figure 4(b) shows the bandgap as the magnitudes of the attached masses are varied. As the bilayer membrane-type resonators are composed of two single layer resonators, 
the bandgap structures of the single layer metamaterials are also depicted in Figure 4 (c) and Figure 4(d) for comparison. For clarity, the layer of membrane applied with lower tensile stress is denoted as the resonator $m_{R 1}$ and the other is denoted as the resonator $m_{R 2}$.

According to the figures, in the two single layer metamaterial, when the mass magnitude is increased from $2.0 \mathrm{~g}$ to $60.0 \mathrm{~g}$, the bandgap width of $m_{R 1}$ is enlarging from $2.6 \mathrm{~Hz}$ to $9.8 \mathrm{~Hz}$, whilst the bandgap width of $m_{R 2}$ is enlarging from $3.1 \mathrm{~Hz}$ to $11.4 \mathrm{~Hz}$.

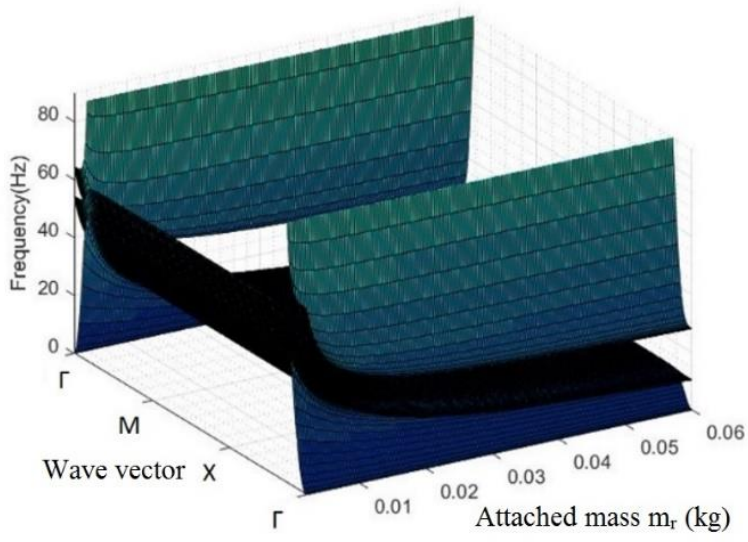

(a)

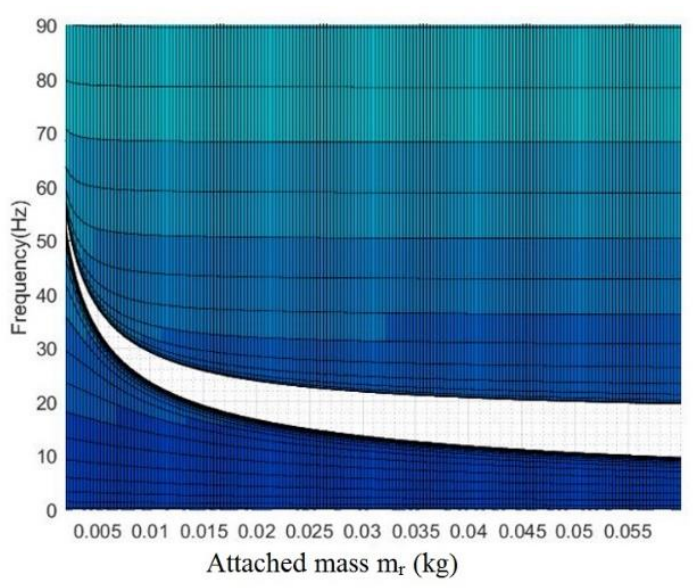

(c)

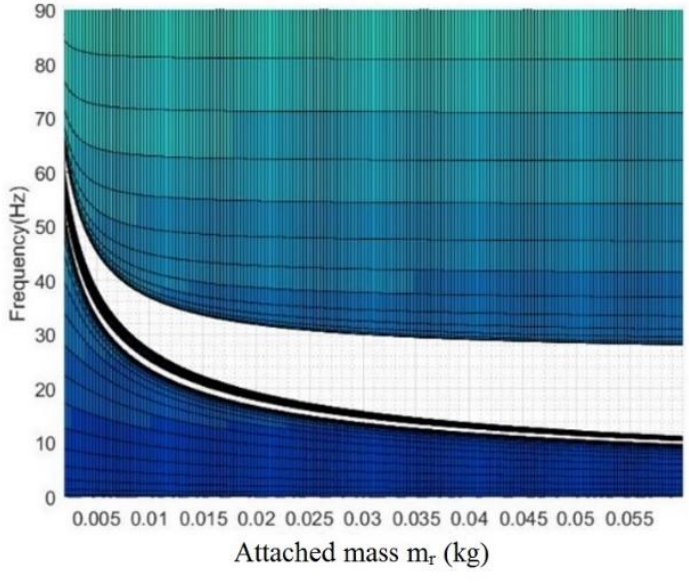

(b)

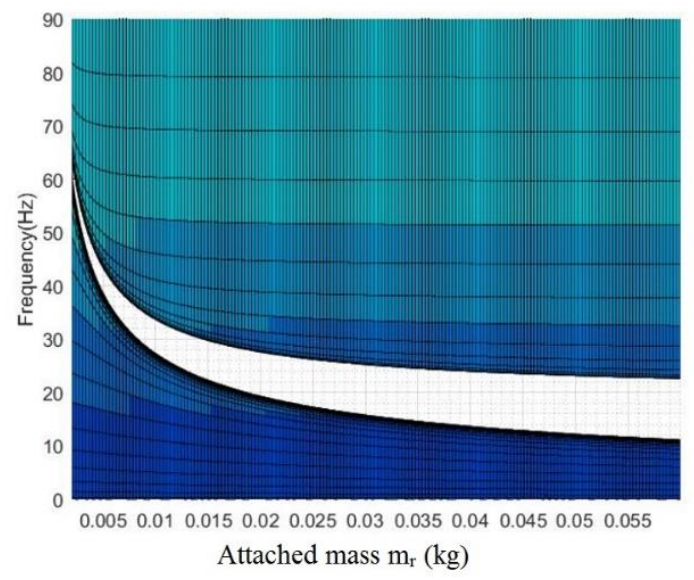

(d)

Figure 4. (a) The band structures of the bilayer metamaterial attached with different mass platelets and (b) the band structures of different mass magnitudes; $(c)$ change of bandgap width of single layer metamaterial applied with $0.6 \mathrm{MPa}$ and (d) 0.8MPa.

In the bilayer one, the first bandgap's lower edge shifts from $52.6 \mathrm{~Hz}$ to $9.7 \mathrm{~Hz}$, and the second bandgap location shifts from $60.7 \mathrm{~Hz}$ to $11.2 \mathrm{~Hz}$. The width of the first bandgap, which is associated with $m_{R 1}$, decreased from $1.7 \mathrm{~Hz}$ to $0.65 \mathrm{~Hz}$. If compared with the corresponding single layer metamaterial, when the mass is increased to $60.0 \mathrm{~g}$, the first bandgap width of bilayer metamaterial is $93.4 \%$ smaller. Meanwhile, the width of the second bandgap increased from $3.8 \mathrm{~Hz}$ to $16.8 \mathrm{~Hz}$, which is about $47.4 \%$ larger than the single layer one. 
Therefore, the bandgap behaviour of bilayer membrane-type resonator is not a simple combination of two independent single layer resonators. In a single layer resonator, the increase of attached mass magnitude will effectively amplify the bandgap width. In a bilayer resonator, however, for fixed membrane stress, the increase of mass magnitude will cause a reduction of the first bandgap width and an increase of the second bandgap width.

It is observed that if the excitation frequency is within $m_{R 1}$ 's bandgap range, the vibration absorption performance can be weakened by the existence of resonator $m_{R 2}$, leading to the narrowing of the first bandgap. On the other hand, if the excitation frequency is within the second bandgap, the absorption capability of the resonator $m_{R 2}$ will be reinforced by the existence of resonator $m_{R 1}$ and the second bandgap is therefore broadened. Naify et al. [38] demonstrated that the increase of mass loaded on the membrane can broaden the frequency bandwidth for sound isolation applications. A similar effect is also found in vibration control applications for the proposed bilayer membrane-type metamaterial as discussed in this section.

\subsubsection{The tensile stress of the membrane-type resonator}

Figure 5 exhibits the change of bandgaps caused by the tuning of tensile stress applied to the membranes. $2.0 \mathrm{~g}$ and $4.0 \mathrm{~g}$ mass platelets are attached to the upper and lower layer of membranes respectively. The tuning range of the applied stress on membranes is from 0.4MPa to 12.0MPa. As the magnitudes of attached masses are kept constant, the increased tensile stress will lead to higher equivalent stiffness and therefore the bandgap location will shift to a higher frequency.

In the bilayer resonator, the first bandgap's starting edge changes from $30.5 \mathrm{~Hz}$ to $166.7 \mathrm{~Hz}$, while the second bandgap's starting edge increases from $42.9 \mathrm{~Hz}$ to $234.7 \mathrm{~Hz}$. Moreover, the first bandgap is produced by the membrane layer with heavier mass $(4 \mathrm{~g})$. Meanwhile, in the bilayer case, the first bandgap width increases from $2.6 \mathrm{~Hz}$ to $14.3 \mathrm{~Hz}$ and the second one changes from $2.4 \mathrm{~Hz}$ to $13.5 \mathrm{~Hz}$, respectively. Similar to the results in Section 2.4.1, when the tensile stress is $12.0 \mathrm{MPa}$, the first bandgap is $18.7 \%$ smaller than the corresponding single layer resonator's bandgap width. The second bandgap is $18.2 \%$ larger than the $0.4 \mathrm{MPa}$ case when the tensile stress is increased to12.0MPa.

As shown in Figure 5(c) and (d), when the stress is increased to 12.0MPa, the bandgap width of the single layer resonator attached with $2.0 \mathrm{~g}$ mass rises from $2.2 \mathrm{~Hz}$ to $12.1 \mathrm{~Hz}$. In addition, the other single layer resonator's bandgap width rises from $3.0 \mathrm{~Hz}$ to $16.6 \mathrm{~Hz}$. So the increase in tensile stress can enlarge the bandgaps.

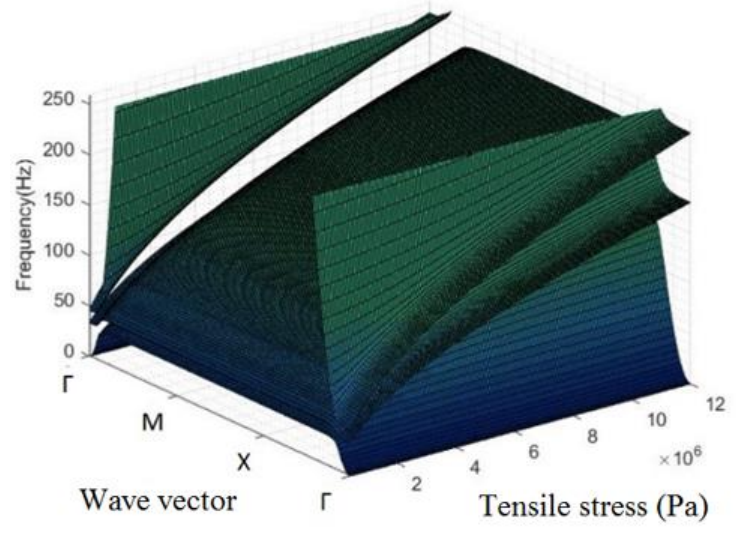

(a)

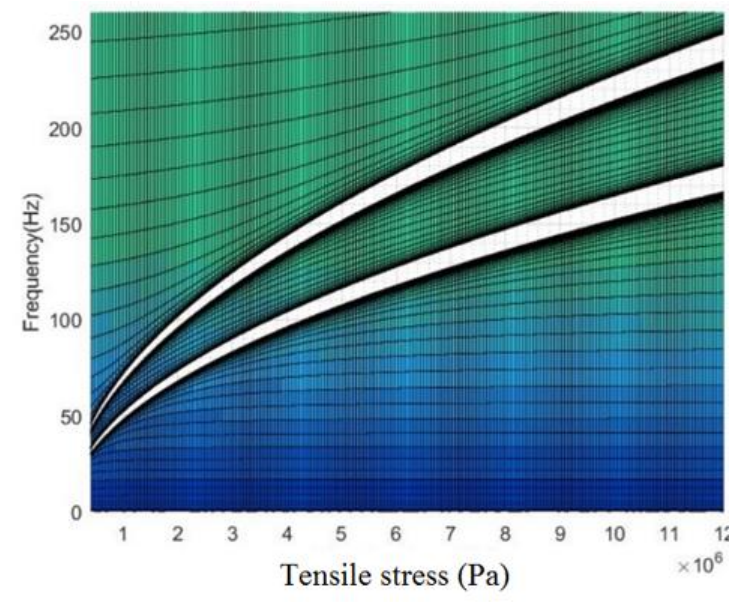

(b) 


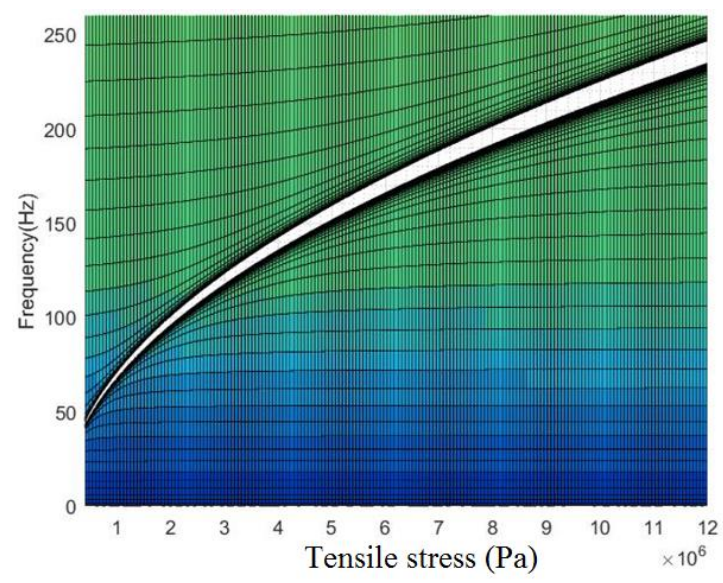

(c)

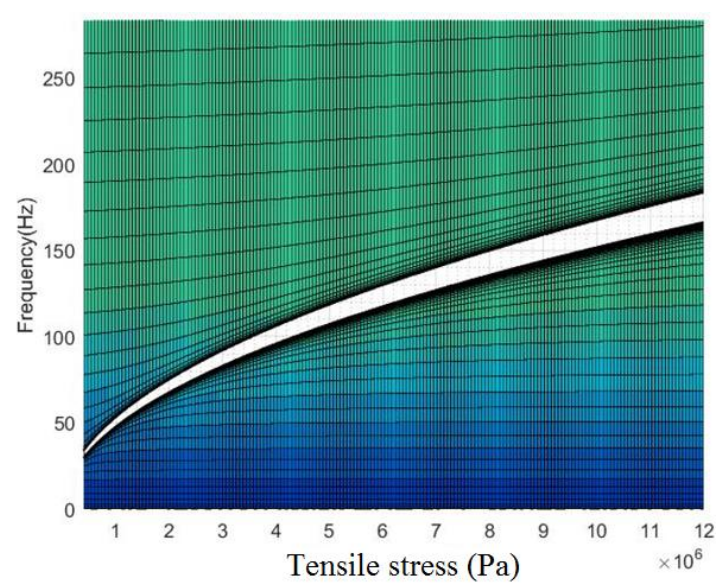

(d)

Figure 5. (a) The band structures of the bilayer metamaterial attached with 2.0g and 4.0g mass; (b) bilayer metamaterial's bandgaps vary with tensile stress applied to membrane; bandgap of single layer metamaterial attached with (c) $2.0 \mathrm{~g}$ mass and (d) $4.0 \mathrm{~g}$ mass with varying tensile stress.

\subsubsection{The periodicity of membrane-type resonators}

The periodicity of membrane-type metamaterial is defined as the distance between the adjacent membrane-type resonators attached to the plate. In this subsection, the effect of the periodicity of bilayer membrane-type metamaterial is investigated. The range of resonator's periodicity is varied from $0.05 \mathrm{~m}$ to $0.195 \mathrm{~m}$, with a $0.05 \mathrm{~m}$ resolution. The tensile stresses of the two membrane-type resonators are respectively defined as $2.0 \mathrm{MPa}$ and $4.0 \mathrm{MPa}$. The attached mass are $10.0 \mathrm{~g}$ and $5.0 \mathrm{~g}$ respectively.

As shown in Figure 6, the starting frequencies of bandgaps are maintained because the periodicity adjustment is not affecting the resonator's resonant frequency. Otherwise, the increase of the metamaterial's periodicity will significantly decrease the bandgap width. When the periodicity is increased, the bandgap width will decrease. This is because of the smaller number of resonators leads to a lower vibration absorption capacity. Theoretically, when the lattice constant is approaching infinity, it describes an infinite size plate with only one resonator attached so the bandgap will finally disappear.

In practice, the bandgap will disappear when the lattice constant increases to a certain value. Figure 6(b) presents the bandgap change of the same bilayer resonator when the periodicity is adjusted from $0.05 \mathrm{~m}$ to $0.4 \mathrm{~m}$. It is observed that the second bandgap, which is associated with a resonator with smaller mass, disappears when the periodicity is larger than $0.21 \mathrm{~m}$. In addition, the first bandgap disappears at about $0.34 \mathrm{~m}$. 


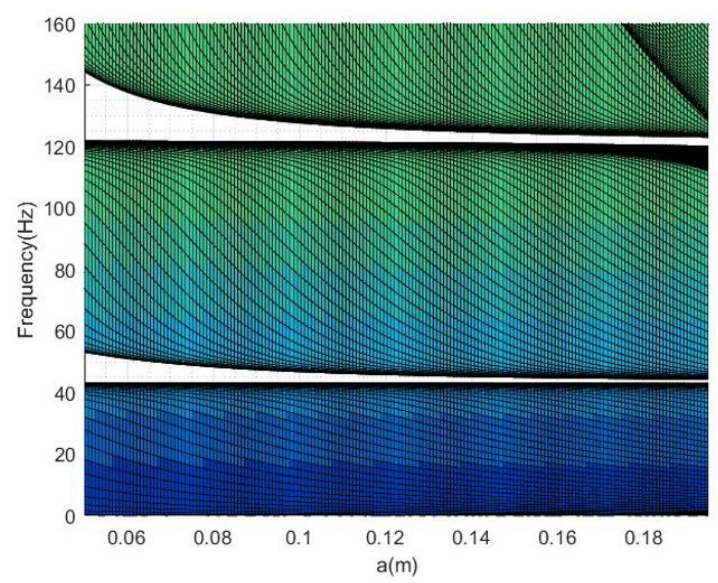

(a)

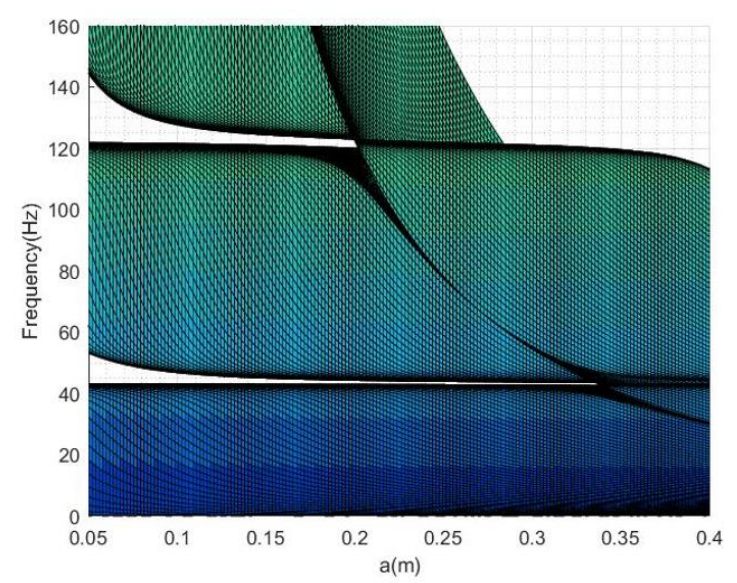

(b)

Figure 6. The change of bandgap when the periodicity of attached resonators, $a$, is tuned from $0.05 m$ to (a) $0.195 \mathrm{~m}$; (b) $0.4 m$.

On the other hand, the magnitudes of attached masses are also closely related to the vibration absorption performance. To demonstrate the effect of attached mass and periodicity on bandgap property, three configurations are taken for comparison. In these cases, the periodicities are set as $60 \mathrm{~mm}, 120 \mathrm{~mm}$ and $240 \mathrm{~mm}$, respectively. In addition, the masses on both membranes are adjusted as $2.0 \mathrm{~g}, 8.0 \mathrm{~g}$ and $32.0 \mathrm{~g}$ correspondingly to ensure the total attached mass in a unit area is the same in three cases. In the meantime, the stress is accordingly adjusted as well to maintain the resonant frequencies unchanged.

Figure 7 presents the bandgap location and widths of these cases with Table 2 describing the details. According to the results, when the periodicity, attached mass and the applied stress are simultaneously increased, both the first and second bandgaps' widths are broadened. In a unit area, the total attached mass of the resonator is the same, yet the one with a larger mass and higher tensile stress will reveal larger bandgap if compared with the counterparts. It demonstrates that for the purpose of forming a broader bandgap, the membrane-type resonator's tensile stress and mass should be designed as large as possible. However, it is also worth noting that the high periodicity will cause a high concentration of mass on the primary structure and may affect its dynamic performance. Therefore, the periodicity should be chosen carefully as there is a compromise between the number of resonators and overall vibration control performance.

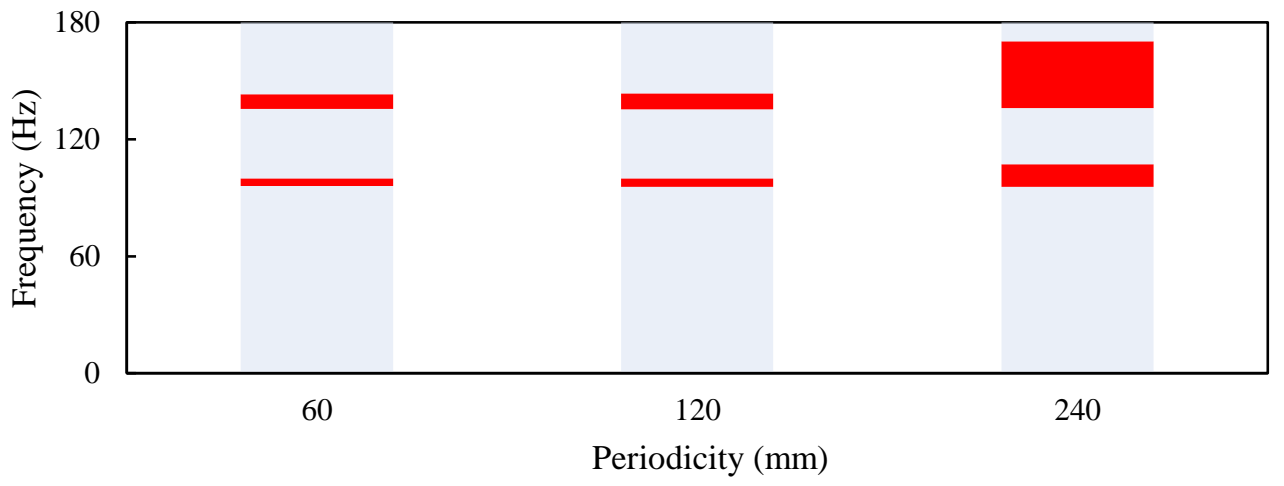

Figure 7. The bandgap location and width for 3 different periodicities. The red areas indicate the bandgap region. 


\begin{tabular}{cccc}
\hline & \multicolumn{3}{c}{ Periodicity } \\
\hline Frequency (Hz) & $60 \mathrm{~mm}$ & $120 \mathrm{~mm}$ & $240 \mathrm{~mm}$ \\
Lower edge 1 & 95.97 & 95.56 & 95.56 \\
Upper edge 1 & 99.95 & 99.87 & 107.05 \\
Band width 1 & 3.98 & 4.31 & 11.49 \\
\hline Lower edge 2 & 135.69 & 135.36 & 135.97 \\
Upper edge 2 & 143.16 & 143.58 & 170.21 \\
Band width 2 & 7.47 & 8.22 & 34.24 \\
\hline
\end{tabular}

Table 2. Data of bandgap location and width for 3 different periodicities.

\subsection{Parametric analysis for bandgap property and optimisation}

The bilayer membrane-type resonator can be optimised by tuning the combination of design parameters. The total bandgap widths of the bilayer resonator with different mass and tensile stress settings are studied in comparison to those of single layer resonators' bandgap widths.

In this case, the mass and tensile stress of the lower layer membrane are respectively defined as: $m_{R 1}=4.0 \mathrm{~g}$ and $T_{1}=1.0 \mathrm{MPa}$. The mass of the upper layer is changed from $0.8 \mathrm{~g}$ to $8.0 \mathrm{~g}$ and tensile stress is changed from $2 \mathrm{MPa}$ to $12.5 \mathrm{MPa}$. The differences of bandgap width between bilayer and single layer resonators are calculated and plotted against the mass ratio $\left(m_{R 2} / m_{R 1}\right)$ and the tensile stress ratio $\left(T_{2} / T_{1}\right)$.

Figure 8(a) presents the first bandgap width difference when the mass ratio and tensile stress ratio are adjusted. A negative value of the bandgap width's difference indicates that the bilayer bandgap width is smaller than the corresponding single layer resonator's bandgap width. As previously mentioned, the first bandgap width is suppressed in a bilayer membrane-type configuration, therefore the values are all negative.

According to Figure 8(b), the increase of mass ratio will further suppress the first bandgap width. When the tensile stress ratio is relatively small, the increase of mass ratio will lead to a rapid decrease of the first bandgap width in the bilayer resonator. This effect is weakened if the tensile stress ratio is higher.

Otherwise, according to Figure 8(c), when the mass ratio is small, the change of tensile stress ratio has no significant effect on the bandgap width. On the contrary, when the mass ratio is relatively large and the tensile stress ratio is increased, the bandgap width difference will decrease rapidly before stabilizing. Thus, if the mass ratio is large, higher tensile stress ratio is recommended in order to reduce the bandgap difference of the first bandgap.

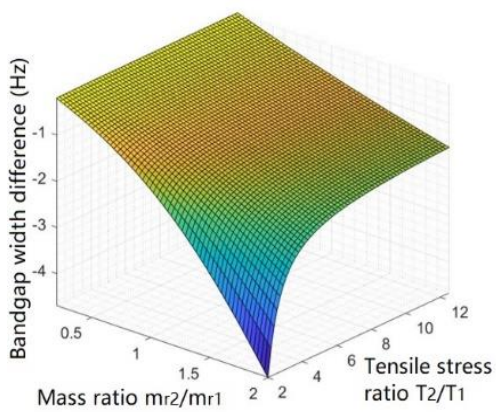

(a)

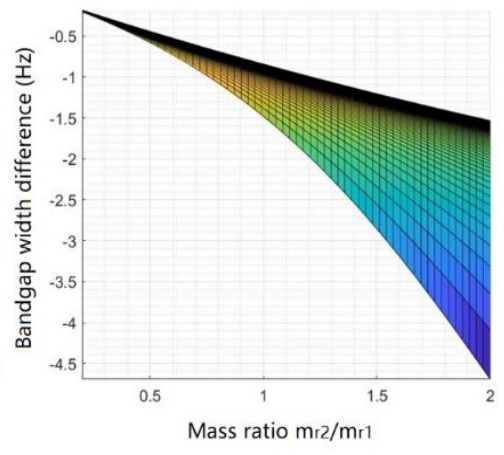

(b)

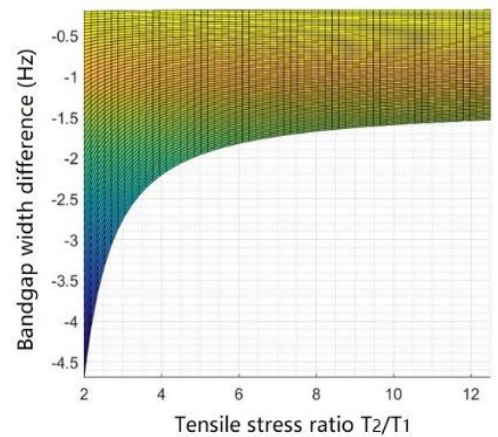

(c)

Figure 8. (a) The lower bound bandgap width difference between bilayer and corresponding single layer resonators; (b) bandgap width difference vs. mass ratio; (c) bandgap width difference vs. tensile stress ratio. 
Figure 9 presents the change of the second bandgap width. As shown in Figure 9(b), in contrast to the first bandgap, the increase of mass ratio will enlarge the second bandgap width of the bilayer resonator. This effect is particularly stronger when the tensile stress ratio is relatively small. In addition, according to Figure 9(c), the change of tensile stress ratio barely has any effect on the bandgap width difference when the small mass ratio is employed. However, when the mass ratio is relatively high, the increase of tensile stress ratio will weaken the second bandgap. Therefore, for the purpose of widening the higher bandgap, the mass ratio should be adjusted to higher value whilst the tensile stress ratio being kept as low as possible.

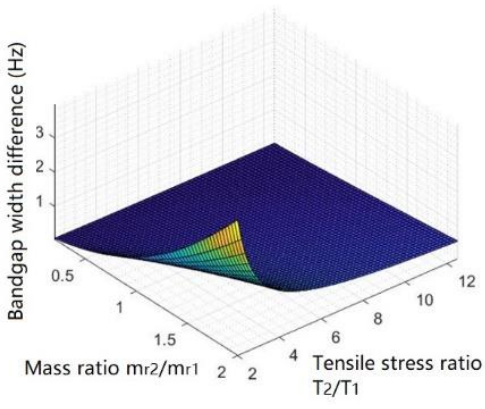

(a)

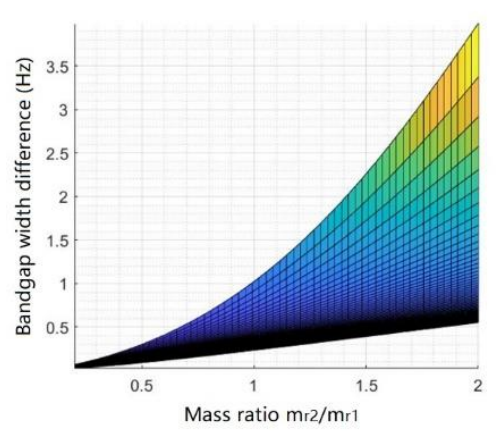

(b)

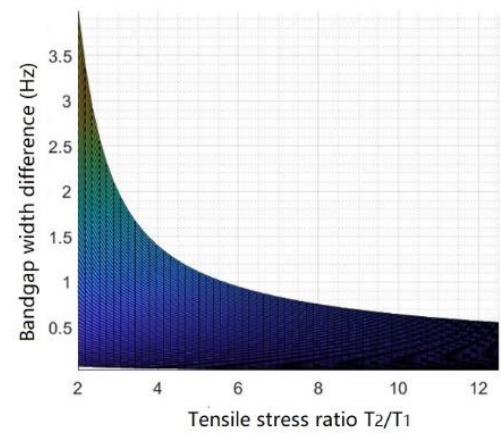

(c)

Figure 9. (a) The upper bound bandgap's width difference between bilayer and corresponding single layer resonators; (b) bandgap width difference vs. mass ratio; (c) bandgap width difference vs. tensile stress ratio.

Figure 10(a) reveals that the total bandgap width difference. As the values are all negative, it indicates that the bilayer resonator's total bandgap width is slightly smaller than the sum of bandgap widths of two single layer resonators. Figure 10(b) illustrates that the increase in the mass ratio will enlarge the bandgap width difference. In addition, when put under the same amount of mass ratio increase, the reduction of bilayer's total bandgap width will be larger if the tensile stress ratio is higher. Otherwise, in accordance with Figure 10(c), the bandgap width difference will change more obviously along with tensile stress ratio when larger mass ratio is applied. In other words, when the mass ratio is small, the adjustment of tensile stress ratio has a smaller effect on the total bandgap width difference. The higher tensile stress ratio will make the total bandgap width of bilayer resonator even smaller.

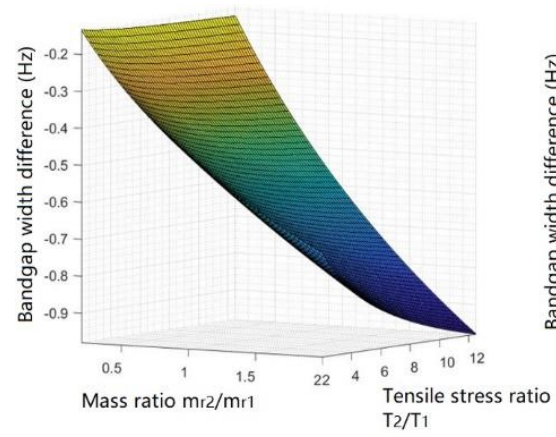

(a)

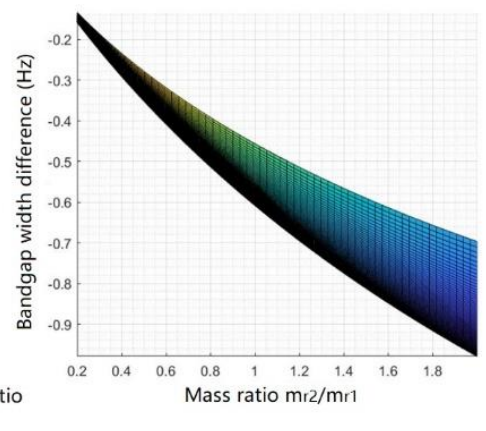

(b)

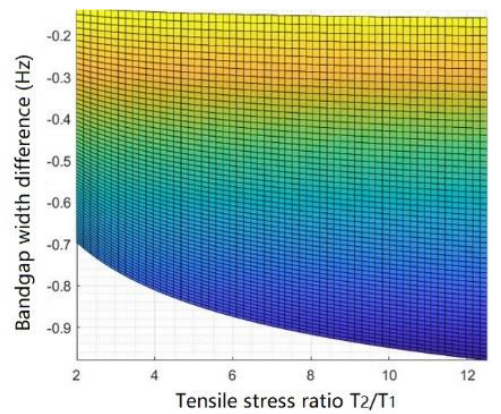

(c)

Figure 10. (a) The total bandgap width difference between bilayer and corresponding single layer resonators; (b) bandgap width difference vs. mass ratio; (c) bandgap width difference vs. tensile stress ratio.

The results provide important design guidelines for the bilayer membrane-type metamaterial. To maintain the first bandgap width of the bilayer membrane-type metamaterial as much as possible, a small mass ratio should be used, and the location of bandgap can be tuned by adjusting the tensile 
stress ratio. By adopting this design parameter combination, the bilayer membrane-type metamaterial's total bandgap width is only slightly suppressed (less than $1 \mathrm{~Hz}$ ).

However, the second bandgap can be widened by adopting a large mass ratio and a low tensile stress ratio. These characteristics allow the bilayer resonator possesses more agile bandgap tuning capability whilst still keeping the same total bandgap width. Moreover, a compromise is needed when choosing different design parameters. The utilisation of bilayer resonators also requires a smaller area than the combined utilisation of two single membrane-type resonators. So the bilayer one can have better application potential and more agile tuning capability.

\section{Simulation studies of a finite bilayer membrane-type metamaterial structure}

In this section, in order to investigate the vibration absorption performance and to verify the accuracy of the modified PWE model, a case study of a finite plate structure with attached bilayer membrane-type metamaterial is conducted. In the proposed model, the accuracy of the estimated fundamental resonant frequency of membrane-type resonator will affect the precision of the bandgap width and location prediction directly. In the proposed theoretical model, the attached mass on the membrane was assumed as a concentrated point mass. In reality, however, the attached mass will occupy a certain area. Therefore, the size of mass will differentiate the shape function from the assumption in the analytical model, and lead to certain error. In this work, the mass is attached as a circular platelet and the radius of the platelet $r_{m}$ is adjusted.

To investigate the influence of mass platelet's radius to the accuracy of the analytical model, a single layer model with varying mass radius $r_{m}$ is constructed. For each radius, the lower edge frequencies of the bandgaps are obtained by the theoretical model $\left(f_{R}\right)$ and then compared with the numerical simulation results $\left(f_{C}\right)$. The applied tensile stress is varied from $0.1 \mathrm{MPa}$ to $1.0 \mathrm{MPa}$ with a step size of $0.1 \mathrm{MPa}$, so the number of data samples is $\mathrm{N}=10$ for each radius. The differences between $f_{R}$ and $f_{C}$ for each radius are averaged, and then plotted against the dimensionless size ratio of the mass radius and membrane side length $r_{m} / L$. The results are shown in Figure 11 . It is found that along with the increase of length ratio, the average error is increasing. This indicates that the larger mass platelet size, the larger is the error. Therefore, in this study, the smallest mass radius of $6 \mathrm{~mm}$ is chosen to keep the error relatively low.

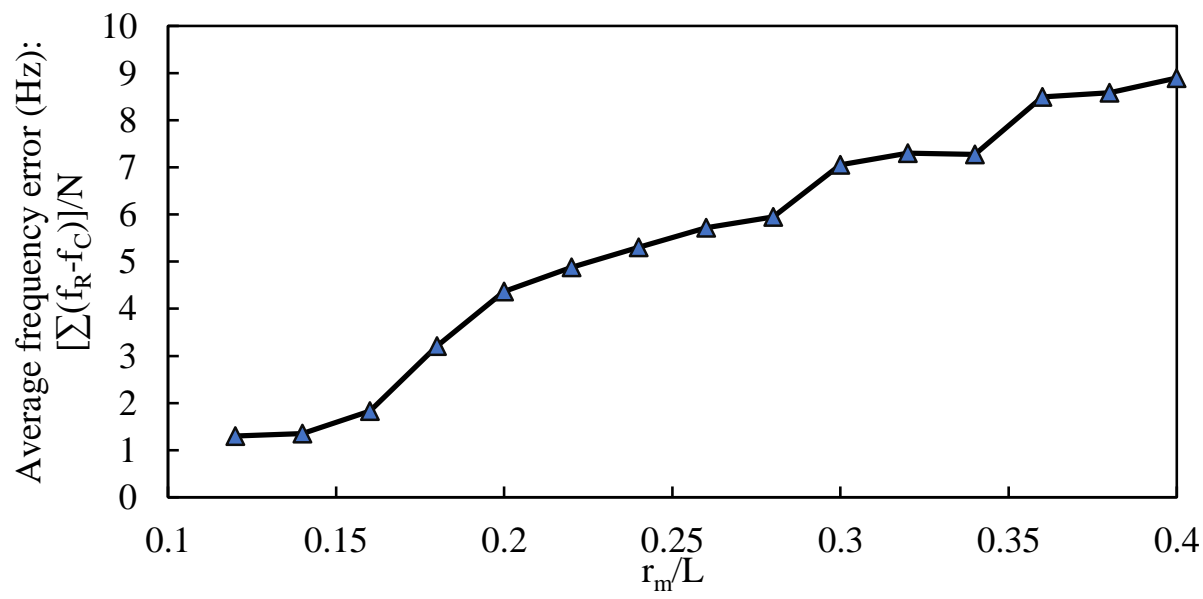

Figure 11. The average frequency errors between the analytical and numerical simulation results. 


\subsection{A finite plate structure with a membrane-type metamaterial}

To examine the bandgap property of a finite plate structure with an attached membrane-type metamaterial, a $240 \times 500 \mathrm{~mm}$ aluminium plate with attached bilayer membrane-type resonators is constructed by using software COMSOL Multiphysics 5.2 as shown in Figure 12. Otherwise, 10\% damping is incorporated into the imaginary part of membrane material's modulus. The left edge of the plate is applied with fixed boundary condition, while the excitation input is applied at the right edge of the structure and pre-stress conditions are applied to the membranes. The acceleration signal is measured at point $A$, while the input acceleration signal is measured from the edge at which the excitation input is applied. The upper layer membrane is applied with 0.6 MPa stress, whilst the lower level membrane is applied with $0.8 \mathrm{MPa}$ stress. Each membrane has a rigid mass of $2.0 \mathrm{~g}$ at its centre. The finite structure model is meshed into tetrahedron elements. The mesh consists of 89,348 domain elements, 76,662 boundary elements, and 13,730 edge elements. The maximum element size is $0.05 \mathrm{~m}$, while the minimum element size is $0.001 \mathrm{~m}$. Considering the highest frequency of interest of $1000 \mathrm{~Hz}$ is associated with the flexural wavelength of about $0.9 \mathrm{~m}$, the mesh size is deemed to be sufficient for obtaining accurate results from the numerical model. Figure 13 shows the frequency response from $30.0 \mathrm{~Hz}$ to $90.0 \mathrm{~Hz}$, with the y-axis described by $F R F=20 \log \left(\frac{\text { Output response }}{\text { Input excitation }}\right)(\mathrm{dB})$.

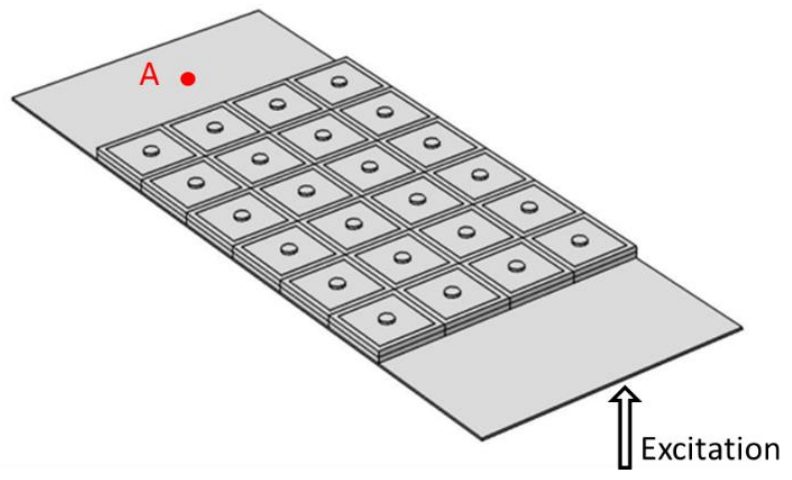

Figure 12. The finite structure of a $4 \times 6$ units of bilayer membrane-type resonators attached to an aluminium plate.

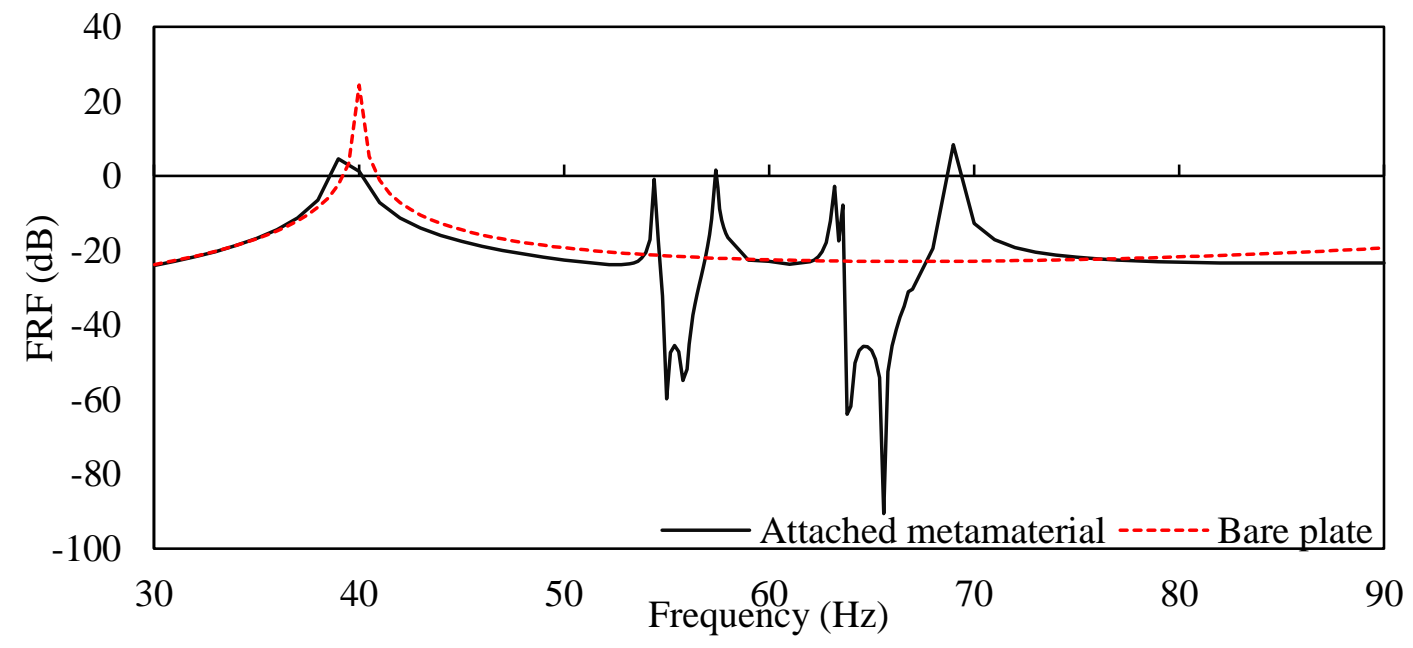

Figure 13. Frequency responses of the bare plate (dashed black) and the plate attached with the bilayer membranetype metamaterial (solid red). 
In Figure 13, the vibration response of the bare thin plate is also presented for comparison. The frequency regions where the FRF curves are lower than the bare plate can be considered as the bandgap frequency regions. The results show that the plate's fundamental resonance has been shifted to a lower frequency because the additional mass that metamaterial contributed to the plate. In addition, there are two bandgaps that appear at frequency ranges of $54.4 \mathrm{~Hz}-57.2 \mathrm{~Hz}$ and $63.2 \mathrm{~Hz}$ $-67.6 \mathrm{~Hz}$. For the analytical model, the obtained bandgaps are located at $52.5-54.3 \mathrm{~Hz}$ and $60.7-$ $64.5 \mathrm{~Hz}$. So the numerical simulation results indicate slightly higher bandgap locations. As indicated in the beginning of Section 4, the size of the attached mass used in the numerical model will lead to differences in the resonant frequency prediction, when compared to the results from the analytical PWE model. However, despite the small differences in bandgap prediction, the bandgap location and width estimation provided by the modified PWE model is consistent with the numerical simulation results, demonstrating the effectiveness of the modelling method.

It should be noted the frequency response in the bandgap region has an asymmetrical shape. This is due to the Fano interference effect that is generated by the periodically allocated resonators [39]. The travelling waves and scattering of the resonant modes of the periodic unit cells will cause the asymmetric dispersion curve. Otherwise, the resonant frequencies of the bare plate are higher than the one attached with membrane-type resonators, because the attached metamaterial increased the system's inertia.

The deformation of the plate at different frequencies are shown in Figure 14. When the incident wave frequency is within the first bandgap range, the first resonant mode of the unit cell will be excited and a significant amount of wave energy will be absorbed and stored within the unit cells. In contrast, when the incident wave frequency is outside the bandgap range, the resonators movement will be mainly in phase with the plate, allowing the wave to propagate through the plate. Figure 14 (c) and Figure 14 (d) presents the two vibration modes that generate bandgaps of the bilayer resonator. It is obvious that in both modes, the resonators are conducting vibration in bending mode.

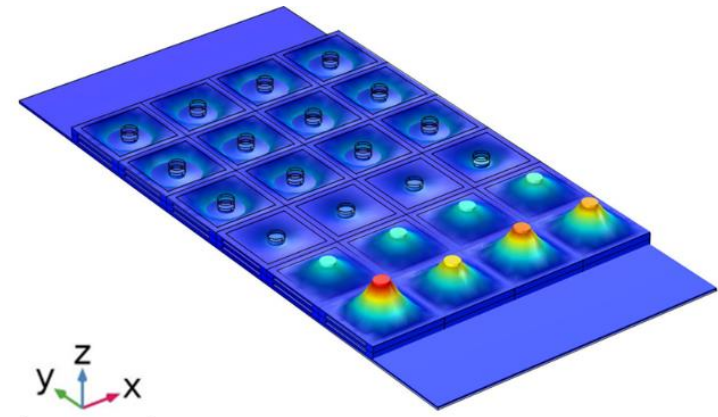

(a)

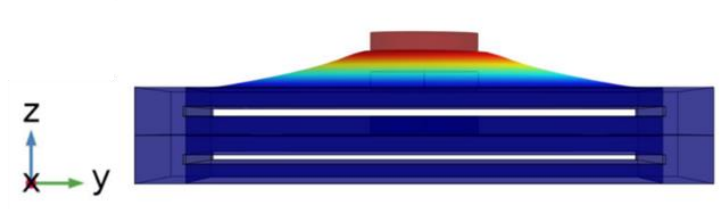

(c)

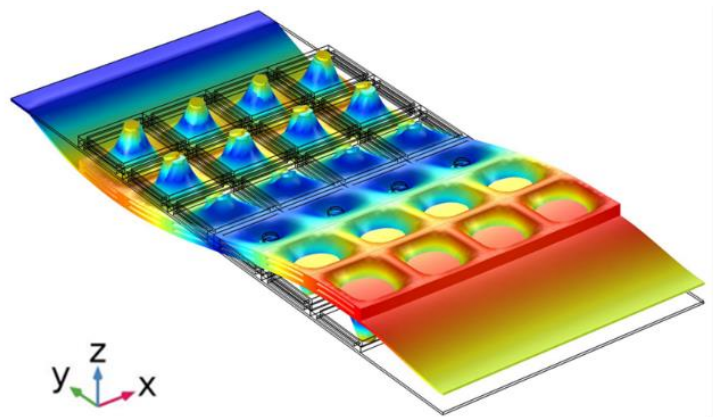

(b)

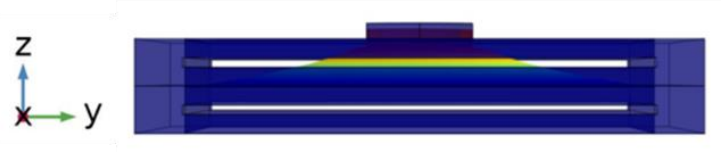

(d)

Figure 14. The deformation of the structure when the incident wave frequency is: (a) within bandgap and (b) outside bandgap; (c) The first and $(d)$ the second vibration mode shapes of the bilayer membrane-type resonators. The attached mass magnitude used is $2 g$. 
To further verify the accuracy of the proposed theoretical model, two other cases are used. Case 1 defines $T_{1}=T_{2}=2.0 \mathrm{MPa}$ and attached mass $m_{R 1}=2.0 \mathrm{~g}$ and $m_{R 2}=5.0 \mathrm{~g}$, respectively. Case 2 defines $T_{1}=2.0 \mathrm{MPa}$ and $m_{R 1}=2.0 \mathrm{~g}$, whilst $T_{2}=4.0 \mathrm{MPa}$ and $m_{R 2}=5.0 \mathrm{~g}$ The corresponding bandgaps obtained through the proposed method and simulation are both presented in Table 3, while the frequency responses are shown in Figure 15.

It is observed that the locations of bandgaps estimated by the proposed analytical method are slightly different with the simulation results but the deviation is within a reasonable range as discussed earlier. In both examples, the second bandgaps, which start from $96.0 \mathrm{~Hz}$, are associated with the resonator with mass $m_{R 1}$. As shown by the results, the second bandgap width will extend when the tensile stress ratio is increased and such results are consistent with the prediction described in Section 3.3.

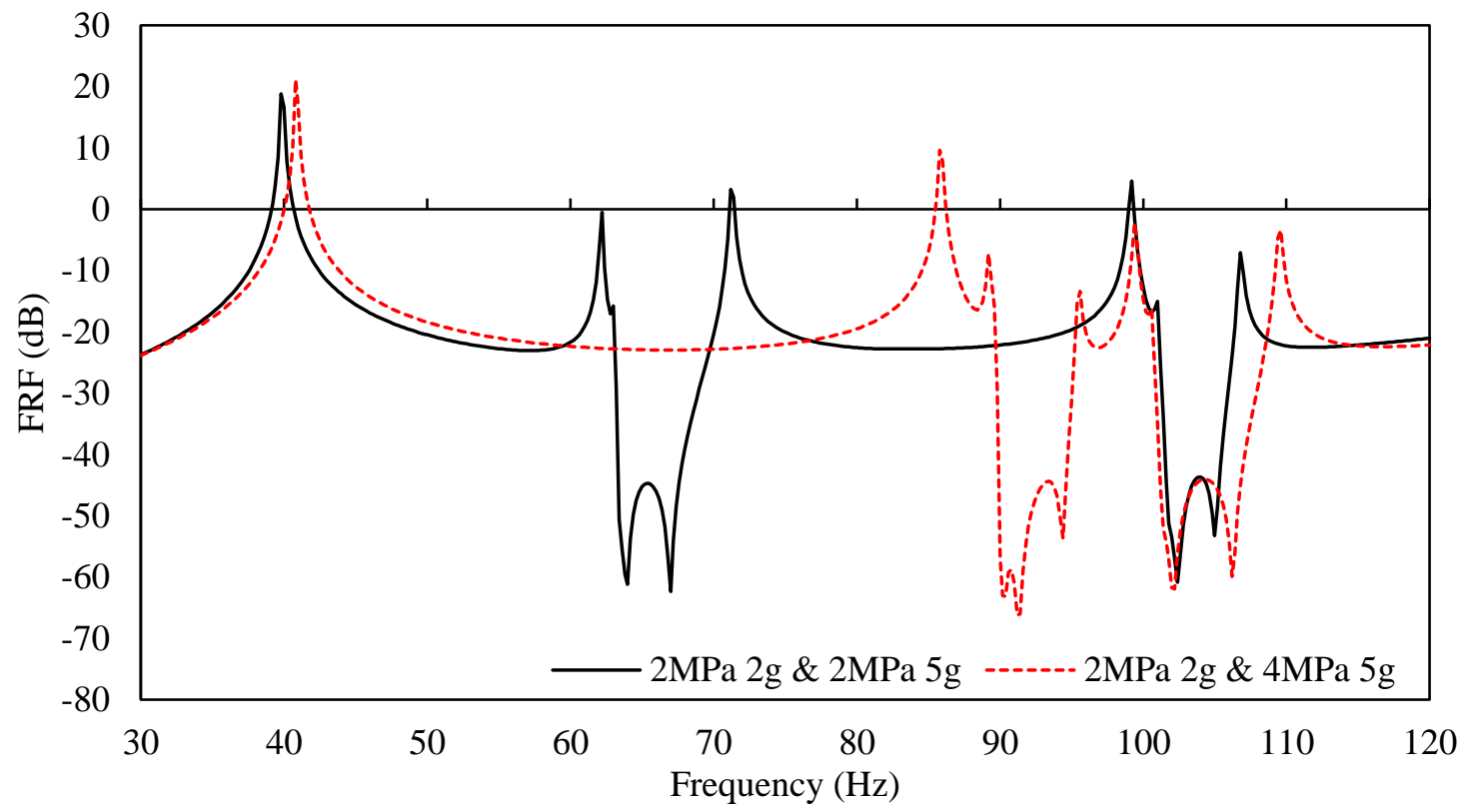

Figure 15. The frequency responses of the plate attached with bilayer membrane-type resonators with different stress and mass magnitudes.

\begin{tabular}{|c|c|c|c|c|c|}
\hline & & \multicolumn{2}{|c|}{ Case 1} & \multicolumn{2}{|c|}{ Case 2} \\
\hline & & Modified PWE & Simulation & Modified PWE & Simulation \\
\hline \multirow{3}{*}{ Bandgap 1} & Lower edge $(\mathrm{Hz})$ & 61.0 & 62.0 & 86.3 & 89.2 \\
\hline & Upper edge (Hz) & 67.2 & 68.0 & 91.4 & 94.0 \\
\hline & Width $(\mathrm{Hz})$ & 6.2 & 6.0 & 5.1 & 4.8 \\
\hline \multirow{3}{*}{ Bandgap 2} & Lower edge $(\mathrm{Hz})$ & 96.0 & 101.0 & 96.0 & 99.8 \\
\hline & Upper edge (Hz) & 101.6 & 104.0 & 105.7 & 106.8 \\
\hline & Width (Hz) & 5.6 & 3.0 & 9.7 & 7.0 \\
\hline
\end{tabular}

\subsection{Effects of the periodicity adjustment of membrane-type resonators}

In Section 4.1, the periodicity of the membrane-type resonator is defined as the outer side length of the frame, so the resonators are adjacent and their frames are connected to each other. In this section, however, the periodicity is adjusted differently to construct different membrane-type 
metamaterial configurations as indicated in Section 3.2.3, and the corresponding bandgap property is studied.

Configurations of membrane-type resonators allocated in (1) $60 \mathrm{~mm}$ periodicity and (2) $120 \mathrm{~mm}$ periodicity are investigated. In the $60 \mathrm{~mm}$ case, the mass and tensile stress settings are the same as in Section 4.1. Otherwise, when the periodicity is increased to $120 \mathrm{~mm}$, the mass magnitude is increased from $2.0 \mathrm{~g}$ to $8.0 \mathrm{~g}$ to achieve an identical total attached mass to the primary structure. Thus, in both finite structures, the total mass attached is $96.0 \mathrm{~g}$ assuming the mass of membranes and frames are negligible. Also, as the attached mass is increased, the tensile stress applied to the membranes is adjusted correspondingly to maintain identical resonant frequencies.

According to Figure 16, the bandgap widths in both cases are similar to each other. The bandgaps for the $120 \mathrm{~mm}$ periodicity case are located at $54.4 \mathrm{~Hz}-58.0 \mathrm{~Hz}$ and $63.6 \mathrm{~Hz}-70.2 \mathrm{~Hz}$. With the same setting parameters, the proposed theoretical model estimates the bandgaps at $52.5 \mathrm{~Hz}-54.3 \mathrm{~Hz}$ and $60.5 \mathrm{~Hz}-64.4 \mathrm{~Hz}$. Similar to the results in Section 4.1, the estimated bandgap locations by numerical simulation are slightly higher than the theoretical model results. Therefore, the estimation of the modified PWE method can also provide effective bandgap estimation for the bilayer membrane-type metamaterial with different levels of periodicity. It should also be noted that the case with $60 \mathrm{~mm}$ periodicity has a higher resonant peak (at about $41.2 \mathrm{~Hz}$ ) than the $120 \mathrm{~mm}$ case $(36.0 \mathrm{~Hz})$. In the metamaterial with $120 \mathrm{~mm}$ periodicity, its unit cells are not connected to each other due to a larger periodic distance as shown in the inset of Figure 16. In contrast, for the metamaterial with $60 \mathrm{~mm}$ periodicity, the unit cells are connected to each other, and therefore increasing the overall stiffness of the structure. Consequently, the resonant frequency is shifted to the higher frequency region.

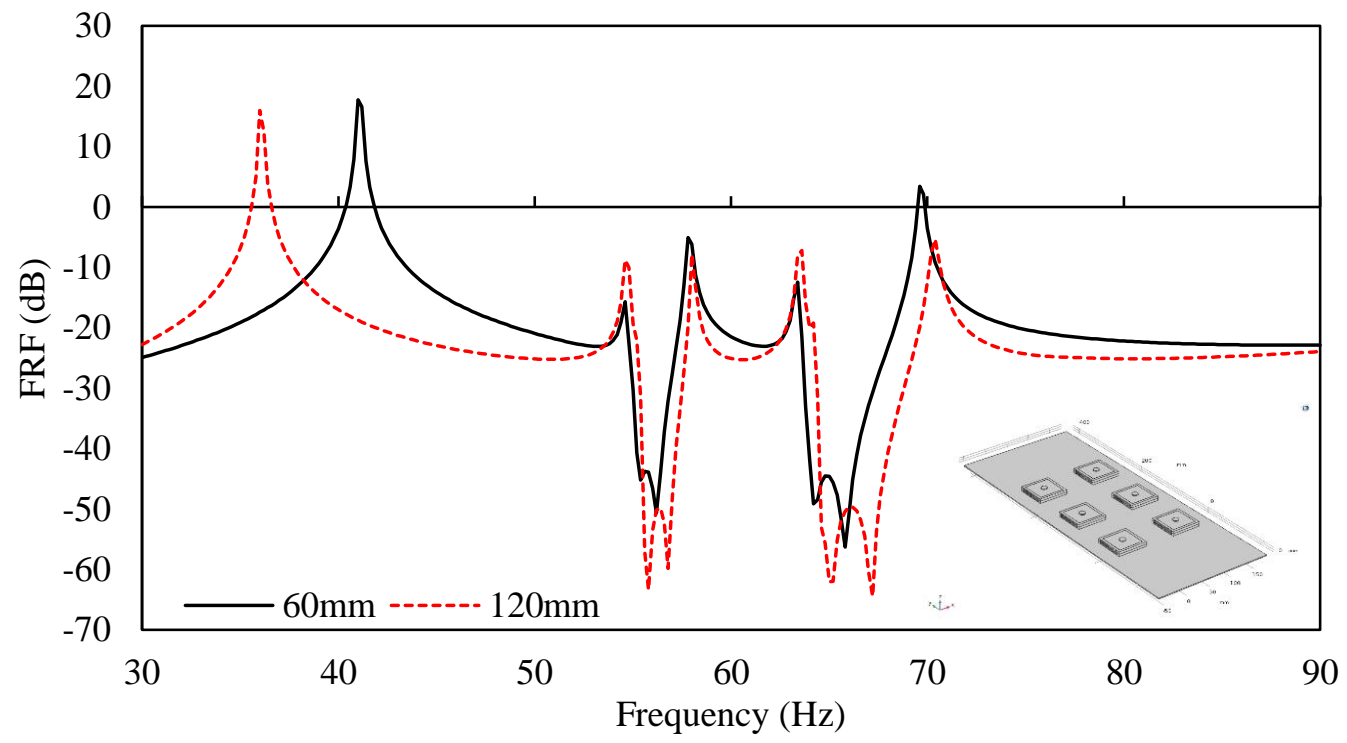

Figure 16. The frequency responses of the metamaterial plate with $60 \mathrm{~mm}$ (black dashed) and $120 \mathrm{~mm}$ (red solid) periodicity. The configuration of the structure is given by the inset.

\section{Conclusions}

In this work, the bandgap property of a bilayer membrane-type metamaterial attached to a thin plate structure has been investigated. An analytical model based on the Plane Wave Expansion method combined with the Rayleigh method is developed. It has been demonstrated in this work that the developed model allowed an accurate prediction of the bandgap property of the bilayer 
metamaterial applied on a thin plate structure, and also it can reveal the effects of design parameters on the bandgap property. In particular, the following main results have been observed:

- The increase of mass magnitudes in resonators and applied tensile stresses of membranes in each unit cell can lead to the extension of bandgap width. However, a proper selection of mass magnitudes is required since the increase of mass will also increase the overall mass of the structure.

- The larger periodicity between unit cells can result in the narrowing of the bandgap width. The bandgap will ultimately disappear if the periodicity reaches a particular value. In addition, the first bandgap width of a bilayer membrane-type metamaterial is always suppressed whilst the second width is widened if compared with the corresponding single layer ones.

- A small bilayer membrane-type resonators' mass ratio $\left(m_{r 2} / m_{r 1}\right)$ will result in the largest total bandgap width of the bilayer membrane-type metamaterial. The bandgap locations can be tuned through the adjustment of the tensile stress ratio $\left(T_{2} / T_{1}\right)$ in each unit cell. Such characteristics allow convenient tuning according to different bandgap requirements.

- The accuracy and feasibility of the proposed analytical method are verified by the numerical simulation. Through the utilisation of the analytical method, rapid design and optimisation of bilayer membrane-type metamaterial can be achieved.

In the future work, the proposed analytical modelling method can be extended beyond the bilayer membrane configuration, to allow the study of the bandgap property of a multi-layered membranetype metamaterial. This study will be beneficial for providing insights into the design of a multilayered membrane-type metamaterial to effectively suppress structural vibration at multiple bandgaps.

\section{Acknowledgements}

The authors acknowledge the financial support from the International Doctoral Innovation Centre, Ningbo Education Bureau, Ningbo Science and Technology Bureau, and the University of Nottingham. This work was also supported by the UK Engineering and Physical Sciences Research Council [grant numbers EP/G037345/1 and EP/L016362/1] and the Zhejiang Provincial First-Class Discipline on Materials Science and Engineering.

\section{References}

[1] M. Kushwaha, P. Halevi, L. Dobrzynski and B. Djafari-Rouhani, "Acoustic band structure of periodic elastic composites," Physical Review Letters, vol. 71, no. 13, p. 2022, 1993. [2] J. Li and C. Chan, "Double-negative acoustic metamaterial," Physical Review E, vol. 70, p. 055602, 2004.

[3] F.-L. Li, Y.-S. Wang and C. Zhang, "A BEM for band structure and elastic wave transmission analysis of 2D phononic crystals with different interface conditions," International Journal of Mechanical Sciences, vol. 144, pp. 110-117, 2018.

[4] M. Sigalas and E. Economou, "Band structure of elastic waves in two dimensional systems," Solid State Communications, vol. 86, no. 3, pp. 141-143, 1993.

[5] Y. Huang, J. Li, W. Chen and R. Bao, "Tunable bandgaps in soft phononic plates with spring-mass-like resonators," International Journal of Mechanical Sciences, vol. 151, pp. 300313, 2019.

[6] Z. Liu, X. Zhang, Y. Mao, Y. Zhu, Z. Yang, C. Chan and P. Sheng, "Locally resonant sonic materials," Science, vol. 289, no. 5485, pp. 1734-1736, 2000. 
[7] Y. Xiao, J. Wen and X. Wen, "Flexural wave band gaps in locally resonant thin plates with periodically attached spring-mass resonators," J. Phys. D: Appl. Phys., vol. 45, no. 19, p. 195401, 2012.

[8] J. Hsu and T. Wu, "Efficient formulation for band-structure calculations of twodimensional phononic-crystal plates," Physical Review B, vol. 74, no. 14, p. 144303, 2006.

[9] C. Goffaux, J. Sanchez-Dehesa and P. Lambin, "Comparison of the sound attenuation efficiency of locally resonant materials and elastic band-gap structures," Physical Review B, vol. 70, no. 18, p. 184302, 2004.

[10] S. Li, Y. Dou, T. Chen, J. Xu, B. Li and F. Zhang, "Designing a broad locally-resonant bandgap in a phononic crystals," Physics Letters A, vol. 383, pp. 1371-1377, 2019.

[11] G. Ma and P. Sheng, "Acoustic metamaterials: From local resonances to broad horizons," Science Advances, vol. 2, no. 2, p. e1501595, 2016.

[12] H. Peng and P. Pai, "Acoustic metamaterial plates for elastic wave absorption and structural vibration suppression," International Journal of Mechanical Sciences, vol. 89, pp. 350361, 2014.

[13] N. Sui, X. Yan, T.-Y. Huang, J. Xu and F.-G. Yuan, "A lightweight yet sound-proof honeycomb acoustic metamaterial," Applied Physics Letters, vol. 106, no. 17, p. 171905, 2015.

[14] N. Gao, J. Wu, L. Yu and H. Xin, "Design of radial phononic crystal using annular soft material with low-frequency resonant elastic structures," Physics Letter A, vol. 380, no. 41, pp. 3326-3332, 2016.

[15] Z. Yang, J. Mei, M. Yang, N. Chan and P. Sheng, "Membrane-type acoustic metamaterial with negative dynamic mass," Physical review letters, vol. 101, no. 20, p. 204301, 2008.

[16] F. Langfeldt and W. Gleine, "Membrane- and plate-type acoustic metamaterials with elastic unit cell edges," Journal of Sound and Vibration, vol. 453, pp. 65-86, 2019.

[17] T.-Y. Huang, C. Shen and Y. Jing, "Membrane- and plate-type acoustic metamaterials," J. Acoust. Soc. AM., vol. 139, no. 6, pp. 3240-3250, 2016.

[18] C. Naify, C. Chang, G. McKnigh and S. Nutt, "Scaling of membrane-type locally resonant acoustic metamaterial arrays," Journal of Acoustical Society of America, vol. 132, no. 4, pp. 2784-2792, 2012.

[19] F. Langfeldt, J. Riecken, W. Gleine and O. von Estorff, "A membrane-type acoustic metamaterial with adjustable acoustic properties," Journal of Sound and Vibration, vol. 373, pp. 1-18, 2016.

[20] Z. Yang, H. Dai, N. Chan, G. Ma and P. Sheng, "Acoustic metamaterial panels for sound attenuation in the 50-1000Hz regime," Applied Physics Letter, vol. 96, p. 041906, 2010.

[21] L. Sun, K. Y. Au-Yeung, M. Yang, S. T. Tang and Z. Yang, "Membrane-type resonator as an effective miniaturized tuned vibration mass damper," AIP Advances, vol. 6, p. 085212, 2016.

[22] L. Sun, "Experimental investigation of vibration damper composed of acoutic metamterials," Applied Acoustics, vol. 119, pp. 101-107, 2017.

[23] N. Gao, H. Hou and Y. Mu, "Low frequency acoustic properties of bilayer membrane acoustic metamaterial with magnetic oscillator," Theoretical \& Applied Mechanics Letters, vol. 7, no. 4, pp. 252-257, 2017.

[24] F. Ma, M. Huang, Y. Xu and J. Wu, "Bilayer synergetic coupling double negative acoustic metasurface and cloak," Scientific Reports, vol. 8, no. 1, p. 5906, 2017.

[25] P. Pai, H. Peng and S. Jiang, "Acoustic metamaterial beams based on multi-frequency vibration absorbers," International Journal of Mechanical Sciences, vol. 79, pp. 195-205, 2014.

[26] J.-S. Chen, Y.-J. Huang and I.-T. Chien, "Flexural wave propagation in metamaterial beams containing membrane-mass structures," International Journal of Mechanical Sciences, Vols. 131-132, pp. 500-506, 2017.

[27] L. Dong, M. Grissom and F. T. Fisher, "Resonant frequency of mass-loaded membranes for vibration energy harvesting applications," AIMS Energy, vol. 3, no. 3, pp. 344-359, 2015. 
[28] J.-S. Chen and I.-T. Chien, "Dynamic behavior of a metamaterial beam with embedded membrane-mass structures," Journal of Applied Mechanics, vol. 84, pp. 121007-1, 2017.

[29] Y. Chen, G. Huang, X. Zhou, G. Hu and C. Sun, "Analytical coupled vibroacoustic modeling of membrane-type acoustic metamaterials: Plate model," The Journal of the Acoustical Society of America, vol. 136, no. 6, pp. 2926-2934, 2014.

[30] G. Hu, L. Tang and X. Cui, "On the modelling of membrane-coupled Helmholtz resonator and its application in acoustic metamaterial system," Mechanical Systems and Signal Processing, vol. 132, pp. 595-608, 2019.

[31] S.-K. Lau, Z. Lu, Y. Liu, X. Yu, M. Z. B. Endot and B. C. Khoo, "Experimental investigations of membrane-type acoustic metamterials with eccentric masses for broadband sound isolation," in Internoise 2017 Conference Proceedings, Hong Kong, 2017.

[32] Y. Zhang, J. Wen, Y. Xiao, X. Wen and J. Wang, "Theoretical investigation of the sound attenuation of membrane-type acoustic metamaterials," Physics Letters A, vol. 376, pp. 14891494, 2012.

[33] Y. Cao, Z. Hou and Y. Liu, "Convergence problem of plane-wave expansion method for phononic crystals," Physics Letters A, vol. 327, no. 2-3, pp. 247-253, 2004.

[34] S. Shi, C. Chen and D. W. Prather, "Plane-wave expansion method for calculating band structure of photonic crystal slabs with perfectly matched layers," JOSA, vol. 21, no. 9, pp. 1769$1775,2004$.

[35] N. Gao, Z. Wei, H. Hou and A. Krushynska, "Design and experimental investigation of V-folded beams with acoustic black hole indentations," Journal of the Acoustical Society of America, vol. 145, no. 1, pp. EL79-EL83, 2019.

[36] N. Gao, Z. Wei, R. Zhang and H. Hou, "Low-frequency elastic wave attenuation in a composite acoustic black hole beam," Applied Acoustics, vol. 154, pp. 68-76, 2019.

[37] J. Mei, G. Ma, M. Yang, Z. Yang, W. Wen and P. Sheng, "Dark acoustic metamaterials as super absorbers for low-frequency sound," Nature communications, vol. 3, p. 756, 2012.

[38] C. Naify, C. Chang, G. McKnight and S. Nutt, "Transmission loss of membrane-type acoustic metamaterials with coaxial ring masses," Applied Physics, vol. 110, p. 12490, 2011.

[39] L. Raghavan and S. Phani, "Local resonance bandgaps in periodic media: Theory and experiment," Journal of Acoustic Society America, vol. 134, no. 3, pp. 1950-1959, 2013. 